\title{
BMJ Open Heart failure in patients presenting with dyspnoea to the emergency department in the Asia Pacific region: an observational study
}

\author{
Gerben Keijzers, ${ }^{1,2,3}$ Anne-Maree Kelly, ${ }^{4}$ Louise Cullen, ${ }^{5,6}$ Sharon Klim, ${ }^{7}$ \\ Colin A Graham, ${ }^{8}$ Simon Craig, ${ }^{9,10,11}$ Win Sen Kuan, ${ }^{12,13}$ Peter Jones, ${ }^{14}$ \\ Anna Holdgate, ${ }^{15,16}$ Charles Lawoko, ${ }^{17}$ Said Laribi ${ }^{18}$
}

To cite: Keijzers G, Kelly A-M, Cullen L, et al. Heart failure in patients presenting with dyspnoea to the emergency department in the Asia Pacific region: an observational study. BMJ Open 2017;7:e013812. doi:10.1136/bmjopen-2016013812

- Prepublication history for this paper is available online. To view these files please visit the journal online (http://dx.doi.org/10.1136/ bmjopen-2016-013812).

Received 10 August 2016 Revised 1 November 2016 Accepted 31 January 2017

CrossMark

For numbered affiliations see end of article.

\section{Correspondence to} Dr Gerben Keijzers; Gerben.Keijzers@health.qld. gov.au

\section{ABSTRACT}

Objectives: To describe demographic features, assessment, management and outcomes of patients who were diagnosed with heart failure after presenting to an emergency department (ED) with a principal symptom of dyspnoea.

Design: Planned substudy of the prospective, descriptive cohort study: Asia, Australia and New Zealand Dyspnoea in Emergency Departments (AANZDEM).

Setting: 46 EDs in Australia, New Zealand, Singapore, Hong Kong and Malaysia collected data over 3 72-hour periods in May, August and October 2014.

Participants: Patients with an ED diagnosis of heart failure.

Outcome measures: Outcomes included patient epidemiology, investigations ordered, treatment modalities used and patient outcomes (hospital length of stay (LOS) and mortality).

Results: 455 (14.9\%) of the 3044 patients had an ED diagnosis of heart failure. Median age was 79 years, half were male and $62 \%$ arrived via ambulance. 392 $(86 \%)$ patients were admitted to hospital. ED diagnosis was concordant with hospital discharge diagnosis in $81 \%$ of cases. Median hospital LOS was 6 days (IQR $4-9$ ) and in-hospital mortality was $5.1 \%$. Natriuretic peptide levels were ordered in $19 \%$, with lung ultrasound $(<1 \%)$ and echocardiography $(2 \%)$ uncommonly performed. Treatment modalities included non-invasive ventilation $(12 \%)$, diuretics $(73 \%)$, nitrates $(25 \%)$, antibiotics $(16 \%)$, inhaled $\beta$-agonists $(13 \%)$ and corticosteroids (6\%).

Conclusions: In the Asia Pacific region, heart failure is a common diagnosis among patients presenting to the ED with a principal symptom of dyspnoea. Admission rates were high and ED diagnostic accuracy was good. Despite the seemingly suboptimal adherence to investigation and treatment guidelines, patient outcomes were favourable compared with other registries.

\section{INTRODUCTION}

Shortness of breath is a common and frightening symptom, which often leads patients

\section{Strengths and limitations of this study}

- This is one of the first studies to collect data on patients with heart failure in the Asia Pacific.

- Data were prospectively collected on consecutive patients with a predefined data dictionary and using an online database.

- Dyspnoea as inclusion criteria is novel and patient-centred, but may limit comparison with other heart failure registries.

- The diagnosis of heart failure was based on clinician judgement, with limited echo performed in emergency department, reflecting real-world practice.

to present to emergency departments (EDs). ${ }^{1}$ The recent EuroDEM pilot study found that $22 \%$ of patients had a cardiac cause for their dyspnoea, with heart failure (HF) as the most common diagnosis (personal communication, Laribi, EuroDEM pilot study, MEMC Congress, Marseilles, France, September 2013). Approximately $1-2 \%$ of the adult population in developed countries has $\mathrm{HF}$, with the prevalence rising to $\geq 10 \%$ among patients over the age of $70 .^{2}$ The symptoms of $\mathrm{HF}$ progressively deteriorate over time, and as such it has an important impact on quality of life. Acute decompensation often leads to hospital admission.

The AANZDEM study, ${ }^{3}{ }^{4}$ built on the methodology of the EuroDEM study (as shared by Laribi), aimed to establish how common shortness of breath was as a symptom in the ED patient population and to describe epidemiological features of patients, distribution of investigations and treatments as well as diagnosis and clinical outcomes. This substudy provides a new insight into ED patients with $\mathrm{HF}$, based on their presenting symptom of breathlessness. 
Several HF registries exist and a recent summary paper $^{2}$ highlighted major regional differences in the severity, aetiology, management and outcomes of patients with $\mathrm{HF}$ in clinical trials and registries. A paucity of data collected outside North America and Europe was noted with a call for further data about geographical variation outside these regions.

To address these unanswered questions, Ambrosy et $a l^{2}$ suggested that a (hospital-based) registry is required which is geographically representative and employs consecutive or intermittently consecutive enrolment and captures comprehensive and longitudinal data including hospital course and postdischarge outcomes.

Moreover, leading international organisations have developed guidelines for the assessment and management of patients with acute HF. ${ }^{5}$ Adherence to these evidence-based guidelines is important but often difficult to measure due to lack of accurate data. This study will also be able to provide insight in adherence to existing guidelines.

We aim to describe a cohort of patients attending an ED in the Asia Pacific region with a principal symptom of dyspnoea and an ED diagnosis of HF in terms of demographics, clinical characteristics, investigations ordered, treatments and patient outcomes (final diagnosis, hospital length of stay (LOS) and in-hospital mortality).

\section{METHODS}

This is a planned substudy of the AANZDEM study. The methodology of that study, including definitions of clinical variables, has been published previously. ${ }^{3}{ }^{4}$ In summary, it was a prospective, descriptive cohort study conducted in EDs in Australia, New Zealand, Singapore, Hong Kong and Malaysia of consecutive adult patients presenting to the ED with dyspnoea as a main symptom (eg, includes patients who had chest pain as well as dyspnoea). Data were collected over three 72-hour periods in May, August and October 2014 (autumn, winter and spring in Australasia) and included demographics, comorbidities, mode of arrival, usual medications, prehospital treatment, initial assessment, ED investigations, treatment in the ED, ED diagnosis, disposition from ED, in-hospital outcome and final hospital diagnosis. Participating hospitals also provided data on total ED presentations and admissions (ward or intensive care unit (ICU)) for each data collection window.

This substudy includes the patients in the AANZDEM study with an ED diagnosis of HF. Patients were included if the principle diagnoses entered by the treating clinician in the ED information system or in the (electronic) medical records included HF, decompensated HF, rightsided HF or acute pulmonary oedema. The primary outcomes of interest are the epidemiology, investigations ordered, treatments given and overall outcomes of these patients. Secondary outcomes were geographical variation.

Analysis was by descriptive statistics, comparisons of proportions and measures of associations $\left(\chi^{2} /\right.$ Fisher's exact test). Non-parametric data were compared using the Mann-Whitney U test. Analysis programs included Analyse-It, ${ }^{7}$ Statistical Package for the Social Sciences (V.22) and Vassar stats. ${ }^{8}$ For analysis by region, Australia and New Zealand data were combined due to the small number of New Zealand patients $(\mathrm{N}=28)$ and compared against the nine South East Asian EDs. A formal sample size calculation was not performed, as this is a descriptive study. Reporting complies with the STROBE guidelines. ${ }^{9}$

Human research ethics approvals for the overall study, as well as predefined substudies, were obtained for all sites according to local requirements. In most jurisdictions, patient consent for data collection was not required.

\section{RESULTS}

Forty-six EDs contributed data on 3044 patients. Thirty-three sites were located in Australia, four in New Zealand, four in Hong Kong, three in Singapore and two in Malaysia. In 2014, the study sites had a combined annual ED census of 2886178 patients.

\section{Demographics and comorbidities}

Four hundred and fifty-five patients (14.9\%) had an ED diagnosis of HF. This represents $0.76 \%$ (95\% CI $0.69 \%$ to $0.83 \%$ ) of all $\mathrm{ED}$ attendances in the study periods.

Patient characteristics (overall and by geographical region) are described in table 1 . The overall median age was 79 years (IQR 67-87), half were male and $62 \%$ arrived via ambulance. Patients in South East Asia were younger (median age 73 vs 81 years, $\mathrm{p}<0.001$ ) and less likely to present by ambulance (52\% vs $67 \%, \mathrm{p}=0.002)$. Common comorbidities included hypertension (72\%), previous diagnosis of HF (57\%), ischaemic heart disease (53\%), hyperlipidaemia (47\%), diabetes $(43 \%)$ and previous atrial fibrillation (AF; 40\%).

Patients in South East Asia were significantly less likely to have a history of HF ( $44 \%$ vs $57 \%, \mathrm{p}=0.003$ ), previous AF (30\% vs $40 \%, p=0.004)$, valvular disease $(\mathrm{p}<0.001)$, chronic obstructive pulmonary disease $(\mathrm{COPD} ; \mathrm{p}<0.001)$ or active malignancy $(\mathrm{p}=0.004)$ as comorbidities.

\section{Medications}

Common regular medications patients were prescribed before presentation are also described in table 1 and included: diuretics $(62 \%)$, statins $(52 \%)$, antiplatelet agents $(50 \%)$, $\beta$-blockers $(49 \%)$ and ACE inhibitors or aldosterone receptor antagonists (ACEi/angiotensin receptor blocker (ARB); 48\%). Patients from the South East Asian countries had a significantly lower use of diuretics (54\%) and ACEi/ARB (39\%), in line with the lower proportion of patients with a history of $\mathrm{HF}$ in these countries.

\section{Clinical features}

Examination findings are shown in table 2. Overall, on presentation to ED, 53\% of patients had a systolic blood pressure over $140 \mathrm{~mm} \mathrm{Hg}$ and three-quarters of the 
Table 1 Patient characteristics: overall, by region and by season

\begin{tabular}{|c|c|c|c|c|}
\hline & \multirow{2}{*}{$\begin{array}{l}\text { Overall } \\
\text { Overall ( } N=455)\end{array}$} & \multicolumn{2}{|l|}{ By region } & \multirow[b]{2}{*}{ p Value } \\
\hline & & Australia and NZ (N=312) & SE Asia ( $\mathrm{N}=143)$ & \\
\hline \multicolumn{5}{|l|}{ Demographics } \\
\hline Age (years, median, IQR) & $79(67-87)$ & $81(69-88)$ & $73(63-82)$ & $<0.001$ \\
\hline \multirow[t]{2}{*}{ Gender $(\mathrm{N}, \%$ male, $95 \% \mathrm{Cl})$} & $227 ; 50 \%$ (45\% to $55 \%)$ & $149 ; 48 \%$ ( $43 \%$ to $53 \%)$ & $78 ; 55 \%(47 \%$ to $63 \%)$ & 0.12 \\
\hline & Missing data=1 & Missing data $=0$ & Missing data=1 & \\
\hline \multirow{2}{*}{$\begin{array}{l}\text { Ambulance arrival (N, \%, } \\
95 \% \mathrm{Cl})\end{array}$} & $280 ; 62 \%$ (58\% to $67 \%)$ & $67 \%(62 \%$ to $73 \%)$ & $52 \%(44 \%$ to $60 \%)$ & 0.002 \\
\hline & Missing data $=6$ & Missing data $=6$ & Missing data $=0$ & \\
\hline Comorbidities & $N, \%(95 \% \mathrm{Cl})$ & $N, \%(95 \% C l)$ & $N, \%(95 \% C l)$ & \\
\hline Hypertension & $327 ; 72 \%$ (68\% to $76 \%)$ & $221 ; 71 \%$ (66\% to $76 \%)$ & $106 ; 74 \%$ (66\% to $81 \%)$ & 0.50 \\
\hline & Missing data $=1$ & Missing data $=1$ & Missing data $=0$ & \\
\hline \multirow[t]{2}{*}{ Previous heart failure } & $256 ; 57 \%$ (52\% to $61 \%)$ & $193 ; 62 \%$ (57\% to $67 \%)$ & $63 ; 44 \%$ (36\% to $52 \%)$ & 0.0003 \\
\hline & Missing data $=2$ & Missing data $=2$ & Missing data $=0$ & \\
\hline \multirow[t]{2}{*}{$\mathrm{IHD}$} & $239 ; 53 \%$ (48\% to $57 \%)$ & $161 ; 52 \%$ (46\% to $57 \%)$ & $78 ; 55 \%$ (46\% to $62 \%)$ & 0.58 \\
\hline & Missing data $=1$ & Missing data $=1$ & Missing data $=0$ & \\
\hline \multirow[t]{2}{*}{ Dyslipidaemia } & $212 ; 47 \%$ (42\% to $52 \%)$ & $147 ; 48 \%$ (42\% to $53 \%)$ & $65 ; 45 \%$ (38\% to $54 \%)$ & 0.65 \\
\hline & Missing data $=4$ & Missing data $=4$ & Missing data $=0$ & \\
\hline \multirow{2}{*}{ Diabetes } & $194 ; 43 \%$ (39\% to $48 \%)$ & $131 ; 43 \%$ (37\% to $48 \%$ ) & $63 ; 44 \%$ (36\% to $52 \%)$ & 0.76 \\
\hline & Missing data=4 & Missing data=4 & Missing data $=0$ & \\
\hline \multirow[t]{2}{*}{ Prior AF } & $180 ; 40 \%$ (35\% to $44 \%)$ & $137 ; 44 \%$ (39\% to $50 \%)$ & $43 ; 30 \%$ (23\% to $38 \%)$ & 0.004 \\
\hline & Missing data $=3$ & Missing data $=3$ & Missing data $=0$ & \\
\hline \multirow[t]{2}{*}{ Renal impairment } & $127 ; 28 \%$ (24\% to $33 \%)$ & $86 ; 28 \%$ (23\% to $33 \%)$ & $41 ; 29 \%(22 \%$ to $37 \%)$ & 0.88 \\
\hline & Missing data $=5$ & Missing data $=5$ & Missing data $=0$ & \\
\hline \multirow[t]{2}{*}{ COPD } & $83 ; 18 \%(15 \%$ to $22 \%)$ & $77 ; 25 \%(21 \%$ to $30 \%)$ & $6 ; 4 \%(2 \%$ to $9 \%)$ & $<0.0001$ \\
\hline & Missing data $=5$ & Missing data $=5$ & Missing data $=0$ & \\
\hline \multirow{2}{*}{ Valvular disease } & $69 ; 15 \%(12 \%$ to $18 \%)$ & $61 ; 20 \%(16 \%$ to $25 \%)$ & $6 ; 4 \%(2 \%$ to $9 \%)$ & $<0.0001$ \\
\hline & Missing data=5 & Missing data=5 & Missing data $=0$ & \\
\hline \multirow{2}{*}{ Anaemia } & $57 ; 13 \%(10 \%$ to $16 \%)$ & $34 ; 11 \%(8 \%$ to $15 \%)$ & $23 ; 16 \%(11 \%$ to $23 \%)$ & 0.13 \\
\hline & Missing data $=5$ & Missing data $=5$ & Missing data $=0$ & \\
\hline \multirow[t]{2}{*}{ Smoker } & $35 ; 8 \%(6 \%$ to $11 \%)$ & $27 ; 9 \%(6 \%$ to $12 \%)$ & $8 ; 6 \%$ (3\% to $11 \%)$ & 0.23 \\
\hline & Missing data $=5$ & Missing data $=5$ & Missing data $=0$ & \\
\hline \multirow[t]{2}{*}{ Active malignancy } & $37 ; 8 \%(6 \%$ to $11 \%)$ & $33 ; 11 \%(8 \%$ to $15 \%)$ & $4 ; 3 \%(1 \%$ to $7 \%)$ & 0.004 \\
\hline & Missing data $=4$ & Missing data $=4$ & Missing data $=0$ & \\
\hline \multirow[t]{2}{*}{ Prior PE } & $17 ; 4 \%$ (2\% to $6 \%)$ & $15 ; 5 \%$ (3\% to $8 \%)$ & $2 ; 1 \%(0.4 \%$ to $5 \%)$ & 0.07 \\
\hline & Missing data $=5$ & Missing data $=5$ & Missing data $=0$ & \\
\hline Usual medications & $N, \%(95 \% C l)$ & $N, \%(95 \% C l)$ & $N, \%(95 \% C l)$ & \\
\hline \multirow[t]{2}{*}{ Diuretic } & $280 ; 62 \%$ (57\% to $66 \%)$ & $203 ; 65 \%$ (60\% to $71 \%)$ & $77 ; 54 \%(46 \%$ to $62 \%)$ & 0.02 \\
\hline & Missing data $=2$ & Missing data $=2$ & Missing data $=0$ & \\
\hline \multirow{2}{*}{ Statin } & $237 ; 52 \%$ ( $48 \%$ to $57 \%)$ & $161 ; 52 \%$ (46\% to $57 \%)$ & $76 ; 53 \%(45 \%$ to $61 \%)$ & 0.78 \\
\hline & Missing data $=1$ & Missing data $=1$ & Missing data $=0$ & \\
\hline
\end{tabular}




\begin{tabular}{|c|c|c|c|c|}
\hline & $\begin{array}{l}\text { Overall } \\
\text { Overall ( } \mathrm{N}=455)\end{array}$ & \multicolumn{2}{|l|}{ By region } & p Value \\
\hline Antiplatelet agent & $\begin{array}{l}226 ; 50 \%(45 \% \text { to } 54 \%) \\
\text { Missing data=2 }\end{array}$ & $\begin{array}{l}148 ; 48 \%(42 \% \text { to } 53 \%) \\
\text { Missing data=2 }\end{array}$ & $\begin{array}{l}78 ; 55 \%(46 \% \text { to } 62 \%) \\
\text { Missing data=0 }\end{array}$ & 0.18 \\
\hline$\beta$-blocker & $\begin{array}{l}224 ; 49 \%(45 \% \text { to } 54 \%) \\
\text { Missing data }=2\end{array}$ & $\begin{array}{l}160 ; 52 \%(46 \% \text { to } 57 \%) \\
\text { Missing data }=2\end{array}$ & $\begin{array}{l}64 ; 45 \%(37 \% \text { to } 53 \%) \\
\text { Missing data=0 }\end{array}$ & 0.18 \\
\hline $\begin{array}{l}\text { ACE inhibitor/angiotensin } \\
\text { receptor blocker }\end{array}$ & $\begin{array}{l}217 ; 48 \%(43 \% \text { to } 53 \%) \\
\text { Missing data }=3\end{array}$ & $\begin{array}{l}161 ; 52 \%(47 \% \text { to } 58 \%) \\
\text { Missing data=3 }\end{array}$ & $\begin{array}{l}56 ; 39 \%(32 \% \text { to } 47 \%) \\
\text { Missing data=0 }\end{array}$ & 0.01 \\
\hline Long-acting oral anticoagulant & $\begin{array}{l}127 ; 28 \%(24 \% \text { to } 32 \%) \\
\text { Missing data }=3\end{array}$ & $\begin{array}{l}105 ; 34 \%(29 \% \text { to } 39 \%) \\
\text { Missing data }=3\end{array}$ & $\begin{array}{l}22 ; 15 \%(10 \% \text { to } 22 \%) \\
\text { Missing data }=0\end{array}$ & $<0.0001$ \\
\hline Calcium channel blocker & $\begin{array}{l}112 ; 25 \%(21 \% \text { to } 29 \%) \\
\text { Missing data }=3\end{array}$ & $\begin{array}{l}74 ; 24 \% \text { ( } 20 \text { to } 29 \%) \\
\text { Missing data=3 }\end{array}$ & $\begin{array}{l}38 ; 27 \% \text { ( } 20 \text { to } 34 \%) \\
\text { Missing data=0 }\end{array}$ & 0.54 \\
\hline Inhaled $\beta$-sympathomimetic & $\begin{array}{l}103 ; 23 \%(19 \% \text { to } 27 \%) \\
\text { Missing data=4 }\end{array}$ & $\begin{array}{l}85 ; 28 \%(23 \% \text { to } 33 \% \\
\text { Missing data=4 }\end{array}$ & $\begin{array}{l}18 ; 13 \%(8 \% \text { to } 19 \%) \\
\text { Missing data }=0\end{array}$ & 0.0004 \\
\hline Nitrate & $\begin{array}{l}85 ; 19 \%(16 \% \text { to } 23 \%) \\
\text { Missing data=4 }\end{array}$ & $\begin{array}{l}51 ; 17 \%(13 \% \text { to } 21 \%) \\
\text { Missing data=4 }\end{array}$ & $\begin{array}{l}34 ; 24 \%(18 \% \text { to } 31 \%) \\
\text { Missing data=0 }\end{array}$ & 0.07 \\
\hline Insulin & $\begin{array}{l}66 ; 15 \%(12 \% \text { to } 18 \%) \\
\text { Missing data=4 }\end{array}$ & $\begin{array}{l}46 ; 15 \%(11 \% \text { to } 19 \%) \\
\text { Missing data }=4\end{array}$ & $\begin{array}{l}20 ; 14 \%(9 \% \text { to } 21 \%) \\
\text { Missing data }=0\end{array}$ & 0.79 \\
\hline Aldosterone antagonist & $\begin{array}{l}54 ; 12 \%(9 \% \text { to } 15 \%) \\
\text { Missing data }=3\end{array}$ & $\begin{array}{l}39 ; 13 \%(9 \% \text { to } 17 \%) \\
\text { Missing data }=3\end{array}$ & $\begin{array}{l}15 ; 10 \%(6 \% \text { to } 17 \%) \\
\text { Missing data }=0\end{array}$ & 0.51 \\
\hline Oral corticosteroid & $\begin{array}{l}22 ; 5 \%(3 \% \text { to } 7 \%) \\
\text { Missing data=5 }\end{array}$ & $\begin{array}{l}17 ; 6 \%(3 \% \text { to } 9 \%) \\
\text { Missing data }=5\end{array}$ & $\begin{array}{l}5 ; 4 \%(2 \% \text { to } 8 \%) \\
\text { Missing data }=0\end{array}$ & 0.35 \\
\hline Home oxygen & $\begin{array}{l}16: 4 \%(2 \% \text { to } 6 \%) \\
\text { Missing data=4 }\end{array}$ & $\begin{array}{l}14 ; 5 \%(3 \% \text { to } 7 \%) \\
\text { Missing data }=4\end{array}$ & $\begin{array}{l}2 ; 1 \%(0.5 \% \text { to } 5 \%) \\
\text { Missing data=0 }\end{array}$ & 0.09 \\
\hline
\end{tabular}


Table 2 Examination findings, testing and pathology findings, overall and by region

\section{Overall \\ Overall $(\mathrm{N}=455)$}

By region

Examination findings in ED

Pulse (bpm)

$\mathrm{SBP}(\mathrm{mm} \mathrm{Hg}, \mathrm{IQR})$

Respiratory rate (per min, IQR)

$86(72-102)$

Missing data $=6$

$143(125-166)$

Missing data $=8$

24 (20-28)

Missing data $=4$

Oxygen saturation on air (\%, where known, IQR)

95\% (92-98\%)

Missing data $=113$

Oxygen saturation on oxygen (\%, where known excludes patients with $\mathrm{SpO}_{2}$ on air, IQR)

97\% (95-99\%)

Missing data $=5$

Peripheral oedema (N, \%, 95\% $\mathrm{Cl}$ )

$\mathrm{SBP}>140 \mathrm{~mm} \mathrm{Hg}(\mathrm{N}, \%, 95 \% \mathrm{Cl})$

$\mathrm{SBP}>160 \mathrm{~mm} \mathrm{Hg}(\mathrm{N}, \%, 95 \% \mathrm{Cl})$

$\mathrm{SpO}_{2}<90 \%$, (on air or oxygen) $(\mathrm{N}, \%, 95 \% \mathrm{Cl}$ )

Respiratory rate $>30 / \mathrm{min},(\mathrm{N}, \%, 95 \% \mathrm{Cl})$

Chest findings in ED

Basal crepitations

$301 ; 77 \%(72 \%$ to $81 \%)$

Missing data $=63$

235; $53 \%$ (48\% to $57 \%$ )

Missing data $=8$

$126 ; 28 \%$ (24\% to $33 \%)$

Missing data $=8$

$74 ; 16 \%$ (13\% to $20 \%)$

Missing data $=5$

$76 ; 17 \%(14 \%$ to $21 \%$

Missing data $=4$

N, \% (95\% Cl)

233; $53 \%$ (49\% to $58 \%)$

Missing data $=19$

86 ; $20 \%$ (16\% to $24 \%$ )

Widespread crepitations

Missing data $=19$

N, \% (95\% Cl)

429; $95 \%$ (92\% to $96 \%$ )

Chest radiograph

Missing data $=0$

3; $0.7 \%(0.2 \%$ to $2 \%)$

Missing data $=0$

$10 ; 2 \%$ ( $1 \%$ to $4 \%)$

Missing data $=2$

$393 ; 86 \%$ (83\% to $89 \%$ )

Missing data $=0$

408; $90 \%$ (87\% to $92 \%)$

Missing data $=0$

25 ; $5 \%$ (4\% to $8 \%)$

Missing data $=0$

$62 ; 14 \%$ (11\% to $17 \%)$

Missing data $=0$

SE Asia ( $\mathrm{N}=143)$

p Value

85 (71-102)

Missing data $=4$

141 (122-160)

Missing data $=7$

24 (20-30)

Missing data $=4$

94\% (90-96\%)

Missing data $=76$

97\% (94-99\%)

Missing data $=4$

$204 ; 76 \%$ (71\% to $81 \%)$

Missing data $=45$

$156 ; 52 \%$ (46\% to $57 \%$ )

Missing data $=7$

76 ; $25 \%$ (20\% to $30 \%)$

Missing data $=7$

56 ; $18 \%$ (14\% to $23 \%)$

Missing data $=4$

63 ; $20 \%$ (16\% to $25 \%$ )

Missing data $=4$

N, \% $(95 \% \mathrm{Cl})$

$146 ; 49 \%$ (44\% to $55 \%$ )

Missing data $=15$

67; $23 \%$ (18\% to $28 \%$ )

Missing data $=15$

N, \% (95\% Cl)

292; $94 \%$ (90\% to $96 \%$ )

Missing data $=0$

1 ; $0.3 \%(0.06 \%$ to $2 \%)$

Missing data $=0$

7; $2 \%$ (1\% to $5 \%)$

Missing data $=2$

299; $98 \%$ (96\% to $98 \%$ )

Missing data $=0$

305; 98\% (95\% to $99 \%$ )

Missing data $=0$

25; $8 \%$ (5\% to $12 \%)$

Missing data $=0$

$33 ; 11$ (8\% to $14 \%)$

NT-proBNP

Missing data $=0$

90 (73-103)

Missing data $=2$

144 (128-172)

Missing data $=1$

21 (19-26)

97\% (95-99\%)

Missing data $=37$

98\% (97-100\%)

Missing data $=1$

$97 ; 78 \%$ (70\% to $84 \%)$

Missing data $=18$

79 ; $56 \%$ (47\% to $64 \%$ )

Missing data $=1$

$50,35 \%$ (28\% to $43 \%)$

Missing data $=1$

8; $6 \%$ (3\% to $11 \%)$

Missing data $=1$

$16 ; 12 \%$ (7\% to $17 \%)$

Missing data $=0$

N, \% (95\% Cl)

$87 ; 63 \%$ (54\% to $70 \%)$

Missing data $=4$

$19 ; 14 \%$ (8\% to $20 \%$ )

Missing data $=4$

$\mathrm{N}, \%(95 \% \mathrm{Cl})$

$137 ; 96 \%$ (91\% to $98 \%)$

Missing data $=0$

$1 \%(0.4 \%$ to $5 \%)$

Missing data $=0$

3; $2 \%(0.7 \%$ to $6 \%)$

Missing data $=0$

$94 ; 66 \%$ (58\% to $73 \%$ )

Missing data $=0$

$103 ; 72 \%$ (64\% to $79 \%)$

Missing data $=0$

0 ; $0 \%(0 \%$ to $2.6 \%)$

Missing data $=0$

29 ; $20 \%$ (15\% to $28 \%$ )

Missing data $=0$ 


\begin{tabular}{|c|c|c|c|c|}
\hline & \multirow{2}{*}{$\begin{array}{l}\text { Overall } \\
\text { Overall }(\mathrm{N}=455)\end{array}$} & \multicolumn{2}{|l|}{ By region } & \multirow[b]{2}{*}{ p Value } \\
\hline & & Australia and $\mathrm{NZ}(\mathrm{N}=312)$ & SE Asia ( $\mathrm{N}=143)$ & \\
\hline Natriuretic peptide (either BNP or NT-proBNP) & $87 ; 19 \%(16 \%$ to $23 \%)$ & $58 ; 19 \%$ ( $15 \%$ to $23 \%)$ & $29 ; 20 \%$ ( $15 \%$ to $28 \%$ ) & 0.76 \\
\hline Troponin & $\begin{array}{l}287 ; 63 \%(59 \% \text { to } 67 \%) \\
\text { Missing data }=0\end{array}$ & $\begin{array}{l}204 ; 65 \%(60 \% \text { to } 70 \%) \\
\text { Missing data }=0\end{array}$ & $\begin{array}{l}83 ; 58 \%(50 \% \text { to } 66 \%) \\
\text { Missing data }=0\end{array}$ & 0.16 \\
\hline $\mathrm{C}$ reactive protein & $\begin{array}{l}145 ; 32 \%(28 \% \text { to } 36 \%) \\
\text { Missing data }=0\end{array}$ & $\begin{array}{l}140 ; 45 \%(40 \% \text { to } 50 \%) \\
\text { Missing data }=0\end{array}$ & $\begin{array}{l}5 ; 4 \%(2 \% \text { to } 8 \%) \\
\text { Missing data }=0\end{array}$ & $<0.0001$ \\
\hline Blood gas analysis & $\begin{array}{l}167 ; 37 \%(32 \% \text { to } 41 \%) \\
\text { Missing data }=0\end{array}$ & $\begin{array}{l}145 ; 46 \%(41 \% \text { to } 52 \%) \\
\text { Missing data }=0\end{array}$ & $\begin{array}{l}22 ; 15 \%(10 \% \text { to } 22 \%) \\
\text { Missing data=0 }\end{array}$ & $<0.001$ \\
\hline Glucose & $\begin{array}{l}256 ; 56 \%(52 \% \text { to } 61 \%) \\
\text { Missing data }=0\end{array}$ & $\begin{array}{l}191 ; 61 \%(56 \% \text { to } 66 \%) \\
\text { Missing data }=0\end{array}$ & $\begin{array}{l}65: 45 \%(38 \% \text { to } 54 \%) \\
\text { Missing data=0 }\end{array}$ & 0.002 \\
\hline D-dimer & $\begin{array}{l}11 ; 2 \%(1 \% \text { to } 4 \%) \\
\text { Missing data=0 }\end{array}$ & $\begin{array}{l}11 ; 4 \%(2 \% \text { to } 6 \%) \\
\text { Missing data }=0\end{array}$ & $\begin{array}{l}0 ; 0 \%(0 \% \text { to } 3 \%) \\
\text { Missing data }=0\end{array}$ & 0.04 \\
\hline Lactate & $\begin{array}{l}125 ; 27 \%(24 \% \text { to } 32 \%) \\
\text { Missing data }=0\end{array}$ & $\begin{array}{l}108 ; 35 \%(30 \% \text { to } 40 \%) \\
\text { Missing data }=0\end{array}$ & $\begin{array}{l}17 ; 12 \%(8 \% \text { to } 18 \%) \\
\text { Missing data=0 }\end{array}$ & $<0.0001$ \\
\hline Albumin & $\begin{array}{l}256 ; 56 \%(52 \% \text { to } 61 \%) \\
\text { Missing data }=0\end{array}$ & $\begin{array}{l}221 ; 71 \%(66 \% \text { to } 76 \%) \\
\text { Missing data }=0\end{array}$ & $\begin{array}{l}35 ; 24 \% \text { ( } 18 \% \text { to } 32 \%) \\
\text { Missing data=0 }\end{array}$ & $<0.0001$ \\
\hline Pathology findings & $N, \%(95 \% \mathrm{Cl})$ & $N, \%(95 \% \mathrm{Cl})$ & $N, \%(95 \% \mathrm{Cl})$ & \\
\hline Serum sodium concentration $<130 \mathrm{mmol} / \mathrm{L}$ & $\begin{array}{l}18 ; 4 \%(3 \% \text { to } 6 \%) \\
\text { Missing data=0 }\end{array}$ & $\begin{array}{l}15 ; 5 \% \text { ( } 3 \% \text { to } 8 \%) \\
\text { Missing data=0 }\end{array}$ & $\begin{array}{l}3 ; 2 \%(0.7 \% \text { to } 6 \%) \\
\text { Missing data=0 }\end{array}$ & 0.20 \\
\hline Haemoglobin $<10 \mathrm{mmol} / \mathrm{L}$ & $\begin{array}{l}67 ; 15 \%(12 \% \text { to } 18 \%) \\
\text { Missing data }=0\end{array}$ & $\begin{array}{l}48 ; 15 \%(12 \% \text { to } 20 \%) \\
\text { Missing data=0 }\end{array}$ & $\begin{array}{l}19 ; 13 \% \text { ( } 9 \% \text { to } 20 \%) \\
\text { Missing data=0 }\end{array}$ & 0.57 \\
\hline Serum creatinine $>80 \mu \mathrm{mol} / \mathrm{L}$ & $\begin{array}{l}313 ; 69 \%(65 \% \text { to } 73 \%) \\
\text { Missing data }=0\end{array}$ & $\begin{array}{l}229 ; 73 \%(68 \% \text { to } 78 \%) \\
\text { Missing data }=0\end{array}$ & $\begin{array}{l}84 ; 59 \%(51 \% \text { to } 66 \%) \\
\text { Missing data=0 }\end{array}$ & 0.002 \\
\hline Serum creatinine $>150 \mu \mathrm{mol} / \mathrm{L}$ & $\begin{array}{l}114 ; 25 \%(21 \% \text { to } 29 \%) \\
\text { Missing data }=0\end{array}$ & $\begin{array}{l}80 ; 26 \%(21 \% \text { to } 31 \%) \\
\text { Missing data=0 }\end{array}$ & $\begin{array}{l}34 ; 24 \% \text { ( } 18 \% \text { to } 31 \%) \\
\text { Missing data }=0\end{array}$ & 0.73 \\
\hline Troponin $>99$ th centile for test & $\begin{array}{l}173 ; 38 \%(34 \% \text { to } 43 \%) \\
\text { Missing data }=0\end{array}$ & $\begin{array}{l}\text { 123; } 39 \%(34 \% \text { to } 45 \%) \\
\text { Missing data }=0\end{array}$ & $\begin{array}{l}50 ; 35 \%(28 \% \text { to } 43 \%) \\
\text { Missing data=0 }\end{array}$ & 0.41 \\
\hline
\end{tabular}

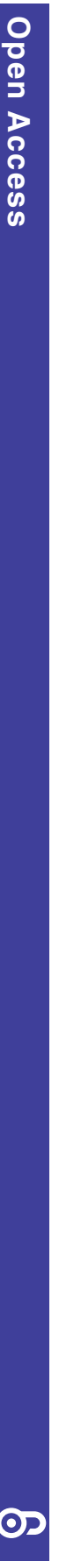

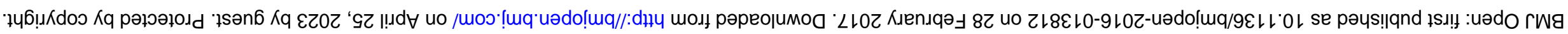




\begin{tabular}{|c|c|c|c|c|}
\hline & \multirow{2}{*}{$\begin{array}{l}\text { Overall } \\
\text { Overall }(\mathrm{N}=455)\end{array}$} & \multicolumn{2}{|l|}{ By region } & \multirow[b]{2}{*}{ p Value } \\
\hline & & Australia and $\mathrm{NZ}(\mathrm{N}=312)$ & SE Asia $(\mathrm{N}=143)$ & \\
\hline Treatment in ED & $N, \%(95 \% \mathrm{Cl})$ & N, \%, (95\% Cl) & $N, \%,(95 \% C l)$ & \\
\hline Oxygen & $285 ; 62 \%$ (58\% to $67 \%)$ & $186 ; 60 \%$ (54\% to $65 \%)$ & $99 ; 69 \%(61 \%$ to 76$)$ & 0.06 \\
\hline Non-invasive ventilation & $56 ; 12 \%(10 \%$ to $16 \%)$ & $49 ; 16 \%(12 \%$ to $20 \%)$ & $7 ; 5 \%(2 \%$ to $10 \% 0$ & 0.002 \\
\hline Mechanical ventilation & $4 ; 1 \%(0.3 \%$ to $2 \%)$ & $2 ; 0.6 \%(0.1 \%$ to $2.3 \%)$ & $2 ; 1 \%(0.4 \%$ to $5 \%)$ & 0.81 \\
\hline \multicolumn{5}{|l|}{ Diuretic } \\
\hline \multirow[t]{2}{*}{ Any } & $332 ; 73 \%(69 \%$ to $77 \%)$ & $236 ; 76 \%(71 \%$ to $81 \%)$ & $96 ; 67 \%(59 \%$ to $74 \%)$ & 0.06 \\
\hline & Missing data $=2$ & Missing data $=2$ & Missing data $=0$ & \\
\hline \multirow{2}{*}{ IV } & $303 ; 67 \%(62 \%$ to $71 \%)$ & $210 ; 68 \%(62 \%$ to $73 \%)$ & $93 ; 65 \%(57 \%$ to $72 \%)$ & 0.65 \\
\hline & Missing data $=2$ & Missing data $=2$ & Missing data $=0$ & \\
\hline \multirow[t]{2}{*}{ Oral } & $46 ; 10 \%(8 \%$ to $13 \%)$ & $40 ; 13 \%(10 \%$ to $17 \%)$ & $6 ; 4 \%(2 \%$ to $9 \%)$ & 0.007 \\
\hline & Missing data $=2$ & Missing data $=6$ & Missing data $=0$ & \\
\hline \multicolumn{5}{|l|}{ Nitrate } \\
\hline \multirow[t]{2}{*}{ Any } & $115 ; 25 \%(22 \%$ to 30$)$ & $72 ; 23 \%$ (19\% to $28 \%)$ & $43 ; 30 \%(23 \%$ to $38 \%)$ & 0.15 \\
\hline & Missing data $=2$ & Missing data $=2$ & Missing data $=0$ & \\
\hline \multirow[t]{2}{*}{ IV bolus } & $1 ; 0.2 \%(0.04 \%$ to $1 \%)$ & $1 ; 0.3 \%(0.06 \%$ to $2 \%)$ & $0 ; 0 \%(0 \%$ to $3 \%)$ & 0.69 \\
\hline & Missing data $=2$ & Missing data $=2$ & Missing data $=0$ & \\
\hline \multirow[t]{2}{*}{ IV infusion } & $31 ; 7 \%(5 \%$ to $10 \%)$ & $16 ; 5 \%$ (3\% to $8 \%)$ & $15 ; 10 \%(6 \%$ to $17 \%)$ & 0.06 \\
\hline & Missing data $=3$ & Missing data $=3$ & Missing data $=0$ & \\
\hline \multirow[t]{2}{*}{ Sublingual/transdermal/oral } & $89 ; 20 \%(16 \%$ to $24 \%)$ & $58 ; 19 \%(15 \%$ to $24 \%)$ & $31 ; 22 \%(16 \%$ to $29 \%)$ & 0.56 \\
\hline & Missing data $=4$ & Missing data $=4$ & Missing data $=0$ & \\
\hline \multirow[t]{2}{*}{ Inotrope/vasopressor } & $5 ; 1 \%(0.5 \%$ to $3 \%)$ & $4 ; 1 \%(0.5 \%$ to $3 \%)$ & $1 ; 0.7 \%(0.1 \%$ to $4 \%)$ & 0.92 \\
\hline & Missing data $=5$ & Missing data $=5$ & Missing data $=0$ & \\
\hline \multirow{2}{*}{ Morphine } & $16 ; 4 \%(2 \%$ to $6 \%)$ & $14 ; 5 \%(3 \%$ to $8 \%)$ & $2 ; 1 \%(0.4 \%$ to $5 \%)$ & 0.16 \\
\hline & Missing data $=6$ & Missing data $=6$ & Missing data $=0$ & \\
\hline \multirow[t]{2}{*}{ Anticoagulant } & $31 ; 7 \%(5 \%$ to $10 \%)$ & $26 ; 8 \%(6 \%$ to $12 \%)$ & $5 ; 4 \%(2 \%$ to $8 \%)$ & 0.08 \\
\hline & Missing data $=5$ & Missing data $=5$ & Missing data $=0$ & \\
\hline \multirow{2}{*}{ Antiplatelet agent } & $40 ; 9 \%(7 \%$ to $12 \%)$ & $28 ; 9 \%(6 \%$ to $13 \%)$ & $12 ; 8 \%(5 \%$ to $14 \%)$ & 0.92 \\
\hline & Missing data $=5$ & Missing data $=5$ & Missing data $=0$ & \\
\hline \multirow[t]{2}{*}{ Antibiotic } & $71 ; 16 \%(13 \%$ to $19 \%)$ & $59 ; 19 \%(15 \%$ to $24 \%)$ & $12 ; 8 \%(5 \%$ to $14 \%)$ & 0.005 \\
\hline & Missing data $=5$ & Missing data $=5$ & Missing data $=0$ & \\
\hline \multirow[t]{2}{*}{ Systemic corticosteroid } & $26 ; 6 \%(4 \%$ to $8 \%)$ & $25 ; 8 \%(6 \%$ to $12 \%)$ & $1 ; 0.7 \%(0.1 \%$ to $4 \%)$ & 0.003 \\
\hline & Missing data $=5$ & Missing data $=5$ & Missing data $=0$ & \\
\hline \multirow[t]{2}{*}{ Inhaled bronchodilator } & $58 ; 13 \%(10 \%$ to $16 \%)$ & $49 ; 16 \%(12 \%$ to $20 \%)$ & $9 ; 6 \%(3 \%$ to $11 \%)$ & 0.007 \\
\hline & Missing data $=5$ & Missing data $=5$ & Missing data $=0$ & \\
\hline Treatment combinations & $N, \%(95 \% \mathrm{Cl})$ & N, \% (95\% Cl) & $N, \%(95 \% C l)$ & \\
\hline \multirow[t]{2}{*}{ Diuretic+nitrate (any route) } & $97 ; 21 \%(18 \%$ to $25 \%)$ & $59 ; 19 \%(15 \%$ to $24 \%)$ & $38 ; 27 \%(20 \%$ to $34 \%)$ & 0.09 \\
\hline & Missing data=2 & Missing data $=2$ & Missing data $=0$ & \\
\hline \multirow[t]{2}{*}{ Diuretic+inhaled bronchodilator } & $47 ; 10 \%(8 \%$ to $14 \%)$ & $41 ; 13 \%(10 \%$ to $18 \%)$ & $6 ; 4 \%(2 \%$ to $9 \%)$ & 0.005 \\
\hline & Missing data $=5$ & Missing data $=5$ & Missing data $=0$ & \\
\hline \multirow{3}{*}{ Diuretic+antibiotic } & $58 ; 13 \%(10 \%$ to $16 \%)$ & $48 ; 16 \%(12 \%$ to $20 \%)$ & $10 ; 7 \%(4 \%$ to $12 \%)$ & 0.02 \\
\hline & Missing data $=5$ & Missing data $=5$ & Missing data $=0$ & \\
\hline & & & & Continued \\
\hline
\end{tabular}




\begin{tabular}{|c|c|c|c|c|}
\hline & \multirow{2}{*}{$\begin{array}{l}\text { Overall } \\
\text { Overall }(\mathrm{N}=455)\end{array}$} & \multicolumn{2}{|l|}{ By region } & \multirow[b]{2}{*}{ p Value } \\
\hline & & Australia and NZ (N=312) & SE Asia ( $N=143)$ & \\
\hline \multirow[t]{2}{*}{ Diuretic+nitrate (any route)+antibiotic } & $18 ; 4 \%(3 \%$ to $6 \%)$ & $15 ; 5 \%(3 \%$ to $8 \%)$ & $3 ; 2 \%(0.7 \%$ to $6 \%)$ & \multirow[t]{2}{*}{0.25} \\
\hline & Missing data $=6$ & Missing data $=6$ & Missing data $=0$ & \\
\hline \multirow{2}{*}{$\begin{array}{l}\text { Diuretic+inhaled bronchodilator+systemic } \\
\text { corticosteroid }\end{array}$} & $13 ; 3 \%(2 \%$ to $5 \%)$ & $13 ; 4 \%(2 \%$ to $7 \%)$ & $0 ; 0 \%(0 \%$ to $3 \%)$ & \multirow[t]{2}{*}{0.03} \\
\hline & Missing data $=5$ & Missing data $=5$ & Missing data $=0$ & \\
\hline \multirow{2}{*}{$\begin{array}{l}\text { Diuretic+inhaled bronchodilator+systemic } \\
\text { corticosteroid+antibiotic }\end{array}$} & $8 ; 2 \%(1 \%$ to $4 \%)$ & $8 ; 3 \%(1 \%$ to $5 \%)$ & $0 ; 0 \%(0 \%$ to $3 \%)$ & \multirow[t]{3}{*}{0.12} \\
\hline & Missing data $=5$ & Missing data $=5$ & Missing data $=0$ & \\
\hline Outcome & $N, \%(95 \% \mathrm{Cl})$ & $N, \%(95 \% \mathrm{Cl})$ & $N, \%(95 \% \mathrm{Cl})$ & \\
\hline \multirow{2}{*}{$\begin{array}{l}\text { Admission to hospital ward (including ICU } \\
\text { and interhospital transfers for admission) }\end{array}$} & $392 ; 86 \%(83 \%$ to $89 \%)$ & $265 ; 85 \%(81 \%$ to $89 \%)$ & $127 ; 89 \%(83 \%$ to $93 \%)$ & \multirow[t]{2}{*}{0.37} \\
\hline & Missing data $=1$ & Missing data $=1$ & Missing data $=0$ & \\
\hline \multirow[t]{2}{*}{ ICU admission } & $19 ; 4 \%(3 \%$ to $7 \%)$ & $13 ; 4 \%(3 \%$ to $7 \%)$ & $6 ; 4 \%(2 \%$ to $9 \%)$ & \multirow[t]{2}{*}{0.81} \\
\hline & Missing data $=1$ & Missing data $=1$ & Missing data $=0$ & \\
\hline \multirow{2}{*}{$\begin{array}{l}\text { ED short stay unit management (excludes } \\
\text { hospital ward admission) }\end{array}$} & $10 ; 2 \%(1 \%$ to $4 \%)$ & $6 ; 2 \%(1 \%$ to $4 \%)$ & $4 ; 3 \%(1 \%$ to $7 \%)$ & \multirow[t]{2}{*}{0.81} \\
\hline & Missing data $=1$ & Missing data $=1$ & Missing data $=0$ & \\
\hline \multirow{2}{*}{$\begin{array}{l}\text { Length of stay for patients admitted to } \\
\text { hospital (days, median, IQR) }\end{array}$} & 6,4 to 9 & 6,3 to 9 & 6,3 to 8 & \multirow[t]{2}{*}{0.44} \\
\hline & Missing data $=22$ & Missing data $=22$ & Missing data $=0$ & \\
\hline \multirow[t]{2}{*}{ Died in ED } & $2 ; 0.5 \%(0.1 \%$ to $2 \%)$ & $2 ; 0.6 \%(0.2 \%$ to $2 \%)$ & $0 ; 0 \%(0 \%$ to $3 \%)$ & \multirow[t]{2}{*}{0.58} \\
\hline & Missing data $=1$ & Missing data $=1$ & Missing data $=0$ & \\
\hline \multirow{2}{*}{$\begin{array}{l}\text { In-hospital mortality for patients admitted to } \\
\text { ward including ICU }\end{array}$} & $20 ; 5 \%$ (3\% to $7 \%)$ & $14 ; 6 \%(3 \%$ to $9 \%)$ & $6 ; 5 \%(2 \%$ to $10 \%)$ & \multirow[t]{2}{*}{0.92} \\
\hline & Missing data $=22$ & Missing data $=22$ & Missing data $=0$ & \\
\hline
\end{tabular}


patients had chest findings of fluid overload on auscultation $(73 \%)$ or peripheral oedema $(77 \%)$. The cohort of South East Asian patients was less likely to have oxygen saturations (on air or oxygen) $<90 \% \quad(6 \%$ vs $18 \%$, $\mathrm{p}=0.007)$ or a respiratory rate $>30 / \mathrm{min}(12 \%$ vs $20 \%$, $\mathrm{p}=0.02$ ) on presentation.

\section{Investigations}

Ordered investigations are shown in table 2. Chest radiograph (chest X-ray) was performed in 95\%, troponin concentration in $63 \%$, blood gas (either arterial or venous) in $37 \%$, $\mathrm{C}$ reactive protein (CRP) in $32 \%$, natriuretic peptide (NP) level (N-terminal pro $\beta$ natriuretic peptide (NT-proBNP) or BNP) in 19\% and D-dimer in $2 \%$. Lung ultrasound $(<1 \%)$ and echocardiography (2\%) were uncommonly conducted in ED. Overall, anaemia (haemoglobin $<10 \mathrm{mmol} / \mathrm{L}, 15 \%$ ) and hyponatraemia (sodium concentration $<130 \mathrm{mmol} / \mathrm{L}, 4 \%$ ) were relatively infrequent findings.

South East Asian sites had significantly lower rates of ordering both routine tests (such as full blood count (72\% vs $98 \%, \mathrm{p}<0.001)$ and electrolytes $(66 \%$ vs $98 \%, \mathrm{p}<0.001)$ ) as well as tests targeting more specific conditions (such as blood gas ( $15 \%$ vs $46 \%, \mathrm{p}<0.001$ ), CRP ( $4 \%$ vs $45 \%$, $\mathrm{p}<0.001)$ and lactate $(12 \%$ vs $35 \%, \mathrm{p}<0.001))$.

\section{Treatment}

Table 3 describes treatment modalities used, which included non-invasive ventilation (12\%), diuretics $(73 \%)$ and nitrates $(25 \%)$. In line with the very low number of patients with a systolic blood pressure $<90 \mathrm{~mm} \mathrm{Hg}$, the use of inotropes was infrequent overall $(<1 \%)$. One hundred and fifty-five (34\%, 95\% CI $30 \%$ to $39 \%)$ patients received antibiotics, inhaled $\beta$-agonists or corticosteroids, suggesting either mixed disease or diagnostic uncertainty. Treatment combinations covering for more conditions than HF alone were less common in South East Asian EDs.

\section{Outcomes}

Outcomes are summarised in table 3. Most patients $(86 \%)$ were admitted to hospital with a median LOS of 6 days (IQR 4-9), including a $4 \%$ admission rate to the ICU. Very few patients (2\%) were admitted to an ED short stay setting. This admission rate pattern, including LOS, was similar across geographic areas. Hospital diagnosis was concordant with ED diagnosis in $81 \%$ of cases, with discordant cases mainly accounted for by hospital discharge diagnosis of COPD (4\%), pulmonary infection $(4 \%)$ and acute coronary syndrome (3\%). In-hospital mortality (including deaths in ED) was $5.1 \%$ (95\% CI $3.3 \%$ to $7.7 \%$ ) and was similar between regions.

\section{DISCUSSION}

\section{Summary}

In the Asia Pacific region, $\mathrm{HF}$ is a common diagnosis in patients presenting to the ED with a principal symptom of dyspnoea with the majority (86\%) of these patients admitted to hospital, a proportion in keeping with other international cohorts. ${ }^{10}$ This unique study of breathless patients in the ED highlights some significant regional differences in the demographic and clinical features on presentation, ordered investigations and treatment of patients with acute HF.

\section{Comparison with existing literature}

The outcomes of patients with HF compare favourably with other registries, despite our cohort being older and compliance with certain features of evidence-based guidelines seemingly suboptimal. The median LOS was 6 days, at the lower end of ranges reported by other studies of between 3.5 and 20 days. ${ }^{11} 12$ In-hospital mortality was $5.1 \%$, at the lower end of the reported median range of $4-30 \% .^{2}$ There was infrequent use of ED short stay admission for this patient group, reflecting an almost dichotomous disposition pathway (inpatient admission vs discharge); however, others have suggested that up to half of the ED patients with HF could be safely discharged after a brief period of observation. ${ }^{13}$ This suggests a missed opportunity in our cohort where short stay wards in ED are usually available. It is possible that a number of inpatient admissions could have been avoided if appropriate local short stay pathways existed.

With a median age of 79 years, patients in our cohort were older than patients in the three largest (US) registries (ADHERE; OPTIMIZE-HF; GWTG-HF 72-74 years) ${ }^{14-17}$ and several of the smaller (European and Japanese) registries (EHFS, ESC-HF, IN-HF, EFICA, RO-AHFS, ATTEND; ranging from 69 to 73 years). ${ }^{2}$ Gender distribution was similar to the three US registries.

Overall patient demographics and comorbidities were comparable with those reported in existing HF registries in the USA and Europe; however, there was considerable regional variation for these variables within our cohort. South East Asian patients were younger than their Australian and New Zealand counterparts; however, they had similar rates of cardiovascular comorbidities (diabetes, ischaemic heart disease, hypertension, dyslipidaemia). Lower rates of pre-existing HF, valvular disease and COPD in the South East Asian patients may be due to their being a median 8 years younger. Our finding is consistent with previous data showing that South East Asian patients with $\mathrm{HF}$ are younger, suggesting that ethnicity may play a part. ${ }^{18}$ Differences in mode of presentation between South East Asian patients and their Australian and New Zealand counterparts, with less ambulance usage in South East Asian patients, may be due to the younger age, fewer comorbidities and lower rates of hypoxia or tachypnoea, or to regional differences in ambulance availability, use and cost. Despite these differences, admission rates and hospital LOS were similar in the different geographical regions.

Our findings of one in five patients overall having an $\mathrm{NP}$ ordered is lower than that expected from acute HF 
guidelines $^{6}$ and difficult to compare to other cohorts due to a lack of data collection or large number of missing data in those cohorts. The National Institute for Health and Care Excellence (NICE) guidelines for acute $\mathrm{HF}^{5}$ recommends using NT-proBNP in patients with suspected new-onset acute HF. Although more than half of our cohort had a previous diagnosis of HF, the overall use of NPs is lower than recommended. It is possible that NPs were mainly used for patients in whom there was diagnostic uncertainty. Some hospitals have test ordering protocols that defer this test ordering to inpatient teams. Using NPs to titrate management in patients with chronic HF is associated with improved mortality, ${ }^{19}{ }^{20}$ but it is unclear if the timing of this measurement matters (ED vs inpatient). Although our study highlights a gap between guidelines and implementation of evidence-based testing in clinical care, it raises a question about the specificity and applicability of these guidelines to our ED setting, since the outcomes of our study compare favourably despite the low NP ordering rates.

There were significant differences in the number of investigations requested for each patient assessed with an ED diagnosis of HF. Overall, patients presenting to Australian EDs had the most pathology tests ordered, with South East Asian EDs the least. This could possibly be explained by the South Asian cohort being younger, having fewer comorbidities, and as such, alternative diagnoses may not have needed to be entertained as frequently. Other regional differences in test ordering could be explained by different levels of perceived medicolegal risk, varying approaches to investigations either clinically or logistically influenced by training programmes, time targets, testing bundles, insurance systems and out-of-pocket expenses. Lower levels of anaemia and hyponatraemia were detected in comparison to other registries and may be due to differences in the chosen cut-offs defining abnormalities (we did not include mild hyponatraemia (130-135 mmol/L)], eg). Overall, over one-third of patients $(37 \%)$ had a blood gas ordered, where approximately one-sixth of patients were hypoxic $(16 \%)$. We cannot comment on whether blood gases were ordered for assessment of ventilation or for other reasons such as the (bedside) assessment of acid-base disorders or electrolyte status.

Three-quarters of patients were treated with diuretics and one-quarter with nitrates, with relatively little geographical variation. Diuretic treatment was less commonly initiated compared with other registries $(73 \%$ vs 76-92\%-ADHERE 92\%, EHFS II-82\%, ATTEND $76 \%$, ALARM 90\% $)^{2}$ and less than guidelines would suggest-since they are recommended as first-line treatment. ${ }^{52}$ The proportion of nitrates used is somewhat more in line with guidelines, which suggest that nitrates should not be routinely offered ${ }^{5}$ or as an adjunct if hypotension is absent. ${ }^{6}$

Despite the seemingly suboptimal adherence to components of the guidelines in terms of investigations (NP ordering) and treatments (diuretic use), patient outcomes are favourable compared with other registries, which raises questions about the utility of the existing guidelines in undifferentiated patients presenting to the ED with dyspnoea. Other treatments and treatment combinations did vary considerably, with South East Asian patients receiving fewer antibiotics, $\beta$-agonists and corticosteroids. As outlined previously, this is possibly a function of this cohort being younger with more commonly single-system disease. Overall, one in six patients received antibiotics $(16 \%)$, which may be a reflection of the proportion of patients being judged by the treating clinician to have mixed disease (including infection), although we are unable to comment on the appropriateness of its use.

Very few $(\sim 1 \%)$ patients received inotropic support compared with registries reporting use between $15 \%$ and $30 \%$ (ADHERE 15\%, EHFS II 30\%, ATTEND 18\%, ALARM 22\%). ${ }^{2}$ This finding suggests regional practice variation that seems unlikely to be due to difference in HF severity (since our cohort was older with similar clinical features and comorbidities). This may be partially explained by the $19 \%$ of patients with a discordant ED and hospital diagnosis. Another possible explanation is that since the patients in our cohort had dyspnoea as a main symptom, they were more likely to have HF with preserved ejection fraction (EF) and, as such, less likely to require inotropic support. In any case, this difference in inotrope use could have implications for interpretations of our outcomes, since even short-term inotrope use has been associated with increased mortality. ${ }^{22}$

\section{Strengths and limitations}

The study sites were located in the South East Asia/ Australasia geographical area and may not be generalisable to other regions. The results of the South East Asia cohort were based on nine sites in three countries (Hong Kong, Singapore and Malaysia), and our finding may not be able to be extrapolated to other countries in South East Asia.

Although we collected data prospectively from consecutive patients presenting with dyspnoea, it is possible that patients with HF were missed due to the design of the overarching study which focused on including patients with dyspnoea as a main symptom. Over $90 \%$ of patients with HF have dyspnoea, ${ }^{23}$ but it is possible that a proportion of patients with HF may have presented without dyspnoea-and rather chest pain, fatigue or peripheral oedema.

The ED diagnosis of HF was based on clinician judgement. Since there is no gold standard for the diagnosis of HF, clinician diagnosis of HF based on the history, signs of fluid overload on physical examination and/or chest radiograph ( \pm response to HF therapy) are often an accepted standard. ${ }^{24}$ The agreement between ED and final hospital diagnosis was $81 \%$, suggesting good accuracy of diagnosis. In some of the discordant cases, where the primary hospital diagnosis was COPD (4\%), 
pulmonary infection $(4 \%)$ or acute coronary syndrome (3\%), HF may have been a secondary process. Targeted use of lung ultrasound ${ }^{25}$ and/or bedside echocardiography and NPs could possibly further improve the diagnostic accuracy in ED. ${ }^{26}$

Echocardiography was rarely performed in the ED, in line with the recent recommendation that urgent echo in the ED is only required when the patient is in cardiogenic shock. ${ }^{27}$ As a result, data on EF were not collected in the vast majority of patients and we cannot comment on proportions of patients with preserved or reduced EF. This makes interpretation regarding adherence to some components of the guidelines related to EF difficult, but it highlights the scope for inclusion of specific ED populations with both preserved and reduced EF in future guidelines.

\section{CONCLUSION}

This study on patients with HF provides a novel insight in several ways, highlighting regional variation in demographics, investigations and treatment in a cohort of patients from a previously under-reported geographical area, the Asia Pacific region. Overall, despite an older cohort, and a seemingly suboptimal adherence to the guidelines in terms of investigations and treatments, patient outcomes are favourable compared with other registries.

\section{Author affiliations}

${ }^{1}$ Department of Emergency Medicine, Gold Coast University Hospital, Gold Coast, Queensland, Australia

${ }^{2}$ School of Medicine, Bond University, Gold Coast, Queensland, Australia

${ }^{3}$ School of Medicine, Griffith University, Gold Coast, Queensland, Australia

${ }^{4}$ Joseph Epstein Centre for Emergency Medicine Research @ Western Health,

Sunshine Australia and Faculty of Medicine, Dentistry and Health Sciences,

The University of Melbourne, Parkville, Victoria, Australia

${ }^{5}$ Department of Emergency Medicine, Royal Brisbane and Women's Hospital, Herston, Queensland, Australia

${ }^{6}$ Faculty of Medicine, University of Queensland and Faculty of Heath, Queensland University of Technology, Herston, Queensland, Australia

${ }^{7}$ Joseph Epstein Centre for Emergency Medicine Research @ Western Health,

Sunshine, Queensland, Australia

${ }^{8}$ Chinese University of Hong Kong, Prince of Wales Hospital, Shatin, Hong Kong

${ }^{9}$ Department of Emergency, Monash Medical Centre, Clayton, Victoria, Australia

${ }^{10}$ School of Clinical Sciences, Monash University, Clayton, Australia

${ }^{11}$ Murdoch Children's Research Institute, Parkville, Victoria, Australia

${ }^{12}$ Department of Emergency Medicine, National University Health System,

Singapore, Singapore

${ }^{13}$ Department of Surgery, Yong Loo Lin School of Medicine, National

University of Singapore, Singapore, Singapore

${ }^{14}$ Department of Emergency Medicine, Auckland City Hospital, Auckland, New Zealand

${ }^{15}$ Department of Emergency Medicine, Liverpool Hospital, Sydney, New South Wales, Australia

${ }^{16}$ University of New South Wales (Southwest Clinical School), Sydney, New

South Wales, Australia

${ }^{17}$ Director, Industry Doctoral Training Centre, ATN Universities, Australia

${ }^{18}$ Department of Emergency Medicine, Tours University Hospital, Paris, France

Acknowledgements AANZDEM Steering Committee: A-MK (Chair), GK (Vice-chair and Queensland), SC (Victoria), CAG (Hong Kong), AH (NSW),
PJ (New Zealand), WSK (Singapore), SL (France). AANZDEM Study Group (includes all hospitals that expressed interest in participation, identified a project lead and had ethics approval): Richard McNulty (Blacktown and Mt Druitt Hospitals NSW), David Lord Cowell (Dubbo Hospital NSW), AH and Nitin Jain (Liverpool Hospital NSW), Tracey de Villecourt (Nepean Hospital NSW), Kendall Lee (Port Macquarie Hospital NSW), Dane Chalkley (Royal Prince Alfred Hospital NSW), Lydia Lozzi (Royal North Shore Hospital NSW), Stephen Asha (St George Hospital NSW), Martin Duffy (St Vincent's Hospital Sydney NSW), Gina Watkins (Sutherland Hospital NSW), David Rosengren (Greenslopes Private Hospital QLD), Jae Thone (Gold Coast Hospital QLD), Shane Martin (Ipswich Hospital QLD), Ulrich Orda (Mt Isa Hospital QLD), Ogilvie Thom (Nambour Hospital QLD), Frances Kinnear and Michael Watson (Prince Charles Hospital QLD), Rob Eley (Princess Alexandra Hospital QLD), Alison Ryan (Queen Elizabeth II Jubilee Hospital QLD), Douglas Morel (Redcliffe Hospital QLD), Jeremy Furyk (Townsville Hospital QLD), Richard Smith (Bendigo Hospital VIC), Michelle Grummisch (Box Hill Hospital VIC), Robert Meek (Dandenong Hospital VIC), Pamela Rosengarten (Frankston Hospital VIC), Barry Chan and Helen Haythorne (Knox Private Hospital VIC), Peter Archer (Maroondah Hospital VIC), SC and Kathryn Wilson (Monash Medical Centre VIC), Jonathan Knott (Royal Melbourne Hospital VIC), Pexter Ritchie (Sunshine Hospital VIC), Michael Bryant (Footscray Hospital VIC), Stephen MacDonald (Armadale Hospital WA), Mlungisi Mahlangu (Peel Health WA), PJ (Auckland City Hospital New Zealand), Michael Scott (Hutt Valley Hospital New Zealand), Thomas Cheri (Palmerston North Hospital New Zealand), Mai Nguyen (Wellington Regional Hospital New Zealand), Colin Graham and Melvin Chor (Prince of Wales Hospital Hong Kong), Chi Pang Wong and Tai Wai Wong (Pamela Youde Nethersole Eastern Hospital Hong Kong), Ling-Pong Leung (Queen Mary Hospital Hong Kong), Chan Ka Man (Tuen Mun Hospital Hong Kong), Ismail Mohd Saiboon (Hospital Universiti Kebangsaan Malaysia, Nik Hisamuddin Rahman (Hospital Universiti Sains Malaysia), Wee Yee Lee (Changi General Hospital Singapore), Francis Chun Yue Lee and Shaun Goh (Khoo Teck Puat Hospital Singapore), WSK (National University Hospital Singapore), SK, Kerrie Russell and A-MK (AANZDEM co-ordinating centre), GK, and SL (steering committee) and CL (Victoria University, statistician).

Contributors GK, A-MK, CAG, SC, PJ, AH and SL were involved in conception and design of the research. GK, SK, WSK, CAG, SC and PJ were involved in acquisition of data. GK, A-MK, LC, WSK, CAG and PJ were involved in analysis and interpretation of the data. GK, A-MK and CL were involved in statistical analysis. GK, A-MK, SK and CAG were involved in obtaining funding and supervising the work. GK, A-MK and LC were involved in drafting the manuscript. GK, A-MK, LC, SK, CAG, SC, WSK, PJ, AH, SL and CL were involved in critical revision of the manuscript for important intellectual content. CL was involved in review of statistical reporting. All authors have approved the final manuscript.

Funding This work was supported by a grant from the Queensland Emergency Medicine Research Foundation, grant number EMPJ-108R21-2014.

Competing interests A-MK reports personal fees from AstraZeneca, MSD, Novartis and Churchill Livingstone, outside the submitted work. A-MK has participated in advisory boards for AstraZeneca, MSD and Novartis and delivered educational sessions sponsored by AstraZeneca, outside the submitted work. LC reports personal fees from Novartis and AstraZeneca, grants and personal fees from Abbott Diagnostics, Alere and Siemens, as well as grants from Roche, outside the submitted work. SL reports personal fees from LILLY and SERVIER, outside the submitted work.

Ethics approval All relevant jurisdictions approved the study.

Provenance and peer review Not commissioned; externally peer reviewed.

Data sharing statement No additional data are available.

Open Access This is an Open Access article distributed in accordance with the Creative Commons Attribution Non Commercial (CC BY-NC 4.0) license, which permits others to distribute, remix, adapt, build upon this work noncommercially, and license their derivative works on different terms, provided the original work is properly cited and the use is non-commercial. See: http:// creativecommons.org/licenses/by-nc/4.0/ 


\section{REFERENCES}

1. Rao AB, Gray D. Breathlessness in hospitalised adult patients. Postgrad Med J 2003;79:681-5.

2. Ambrosy AP, Fonarow GC, Butler J, et al. The global health and economic burden of hospitalizations for heart failure: lessons learned from hospitalized heart failure registries. J Am Coll Cardiol 2014;63:1123-33.

3. Kelly AM, Keijzers G, Klim S, et al. AANZDEM Study Group. Asia, Australia and New Zealand Dyspnoea in Emergency Departments (AANZDEM) study: rationale, design and analysis. Emerg Med Australas 2015;27:187-91.

4. Kelly AM, Keijzers G, Klim S, et al. An observational study of dyspnoea in emergency departments: the Asia, Australia and New Zealand Dyspnoea in Emergency Departments study (AANZDEM). Academic Emerg Med 2016. doi: 10.1111/acem.13118. [Epub ahead of print].

5. Acute heart failure: diagnosis and management. National Institute for Health and Care Excellence. https:// http://www.nice.org.uk/guidance/ cg187 (Last accessed January 2016).

6. McMurray JJ, Adamopoulos S, Anker SD, et al. ESC Committee for Practice Guidelines. ESC guidelines for the diagnosis and treatment of acute and chronic heart failure 2012: the Task Force for the Diagnosis and Treatment of Acute and Chronic Heart Failure 2012 of the European Society of Cardiology. Developed in collaboration with the Heart Failure Association (HFA) of the ESC. Eur J Heart Fail 2012;14:803-69.

7. Analyse-lt. http://analyse-it.com/ (Last accessed January 2016).

8. Vassar stats. http://vassarstats.net/newcs.html (Last accessed January 2016).

9. STROBE statement. Strengthening the reporting of observational studies in epidemiology. http://strobe-statement.org (Last accessed January 2016).

10. Peacock WF. Using the emergency department clinical decision unit for acute decompensated heart failure. Cardiol Clin 2005;23:569-88, viii.

11. Peacock WF, Remer EE, Aponte J, et al. Effective observation unit treatment of decompensated heart failure. Congest Heart Fail 2002;8:68-73.

12. Peacock WF, Emerman CL, on behalf of the PROACTION Study Group. Safety and efficacy of nesiritide in the treatment of decompensated heart failure in observation patients. J Am Coll Cardiol 2003;41:336A.

13. Collins SP, Pang PS, Fonarow GC, et al. Is hospital admission for heart failure really necessary? The role of the emergency department and observation unit in preventing hospitalization and rehospitalization. J Am Coll Cardiol 2013;61:121-6.

14. Yancy CW, Lopatin M, Stevenson LW, et al. ADHERE Scientific Advisory Committee and Investigators. Clinical presentation, management, and in-hospital outcomes of patients admitted with acute decompensated heart failure with preserved systolic function: a report from the Acute Decompensated Heart Failure Nationa Registry (ADHERE) Database. J Am Coll Cardiol 2006;47:76-84.

15. Adams KF Jr, Fonarow GC, Emerman CL, et al. ADHERE Scientific Advisory Committee and Investigators. Characteristics and outcomes of patients hospitalized for heart failure in the United States: rationale, design and preliminary observations from the first 100,000 cases in the Acute Decompensated Heart Failure National Registry (ADHERE). Am Heart J 2005;149:209-16.
16. Gheorghiade M, Abraham WT, Albert NM, et al., for the OPTIMIZE-HF Investigators and Coordinators. Systolic blood pressure at admission, clinical characteristics, and outcomes in patients hospitalized with acute heart failure. JAMA 2006;296:2217-26.

17. Ambardekar AV, Fonarow GC, Hernandez AF, et al. Get With the Guidelines Steering Committee and Hospitals. Characteristics and in-hospital outcomes for nonadherent patients with heart failure: findings from Get With The Guidelines-Heart Failure (GWTG-HF). Am Heart J 2009;158:644-52.

18. Atherton JJ, Hayward CS, Wan Ahmad WA, et al. ADHERE International-Asia Pacific Scientific Advisory Committee. Patient characteristics from a regional multicenter database of acute decompensated heart failure in Asia Pacific (ADHERE International-Asia Pacific). J Card Fail 2012;18:82-8.

19. Savarese G, Trimarco B, Dellegrottaglie S, et al. Natriuretic peptide-guided therapy in chronic heart failure: a meta-analysis of 2,686 patients in 12 randomized trials. PLOS ONE 2013;8: e58287.

20. Felker GM, Hasselblad V, Hernandez AF, et al. Biomarker-guided therapy in chronic heart failure: a meta-analysis of randomized controlled trials. Am Heart J 2009;158:422-30

21. Yancy CW, Jessup M, Bozkurt B, et al. American College of Cardiology Foundation/American Heart Association Task Force on Practice Guidelines. 2013 ACCF/AHA guideline for the management of heart failure: a report of the American College of Cardiology Foundation/American Heart Association Task Force on practice guidelines. Circulation 2013;128:e240-327.

22. Abraham WT, Adams KF, Fonarow GC, et al. In-hospital mortality in patients with acute decompensated heart failure requiring intravenous vasoactive medications: an analysis from the Acute Decompensated Heart Failure National Registry (ADHERE). J Am Coll Cardiol 2005;46:57-64

23. Fonarow GC, Corday E. Overview of acutely decompensated congestive heart failure (ADHF): a report from the ADHERE registry. Heart Fail Rev 2004;9:179-85.

24. Dickstein K, Cohen-Solal A, Filippatos G, et al. ESC Guidelines for the diagnosis and treatment of acute and chronic heart failure 2008: the Task Force for the Diagnosis and Treatment of Acute and Chronic Heart Failure 2008 of the European Society of Cardiology. Developed in collaboration with the Heart Failure Association of the ESC (HFA) and endorsed by the European Society of Intensive Care Medicine (ESICM). Eur Heart J 2008;29:2388-442.

25. Pivetta E, Goffi A, Lupia E, et al. SIMEU Group for Lung Ultrasound in the Emergency Department in Piedmont. Lung ultrasound-implemented diagnosis of acute decompensated heart failure in the ED: a SIMEU multicenter study. Chest 2015;148:202-10.

26. Seronde MF, Laribi S, Collins SP, et al. Heart failure diagnosis in acute conditions has high agreement with inpatient diagnosis. Eur J Emerg Med 2016;23:179-84

27. Mebazaa A, Yilmaz MB, Levy $P$, et al. Recommendations on pre-hospital and early hospital management of acute heart failure: a consensus paper from the Heart Failure Association of the European Society of Cardiology, the European Society of Emergency Medicine and the Society of Academic Emergency Medicine. Eur J Heart Fail 2015;17:544-58. 